\title{
Three year follow up of patients with raised blood pressure identified at health checks in general practice
}

\author{
David Mant, Carolyne McKinlay, Alice Fuller, Tony Randall, Elaine M Fullard, John Muir
}

Imperial Cancer Research Fund General Practice Research Group and University Department of Community Medicine and General Practice, Radcliffe Infirmary, Oxford OX2 6HE

David Mant, MRCGP, research coordinator Alice Fuller, BSC, research officer

Tony Randall, MRCGP, clinical tutor in general practice

John Muir, MD, research fellow

Oxford Centre for

Prevention in Primary

Care, Radcliffe Infirmary, Oxford

Carolyne McKinlay, ALA, research officer

Elaine M Fullard, RGN, facilitator and Oxford project director

Correspondence to: Dr Mant.

\section{Abstract}

Objective-To assess the extent of three year follow up of blood pressure, weight, and smoking habit in patients with raised blood pressure identified at health checks.

Design-Retrospective audit of medical and nursing records.

Setting-Three general practices in Oxfordshire.

Patients -386 Of 448 patients with raised blood pressure (diastolic $\geqslant 90$ or systolic $\geqslant 160 \mathrm{~mm} \mathrm{Hg}$ ) identified from 2935 patients aged 35-64 attending health checks in 1982-4.

Measurements and main results-All records of blood pressure, weight, and smoking habit in the medical record were abstracted for three years after the initial health check. All 42 patients with an initial diastolic blood pressure $\geqslant 105 \mathrm{~mm} \mathrm{Hg}$ and 316 of 344 patients with an initial pressure of $90-104 \mathrm{~mm} \mathrm{Hg}$ had at least one further measurement of their blood pressure. Follow up of smoking habit and of weight was less complete with only half of the 100 smokers and 67 of the 87 obese patients (body mass index $\geqslant 30$ ) having any documented follow up of these risk factors. Annual follow up in the second and third years occurred in $228 / 297(76.8 \%)$ and $232 / 320$ $(72.5 \%)$ in patients with blood pressure $>95 \mathrm{~mm} \mathrm{Hg}$ at the beginning of each year. For patients who smoked annual follow up in these years occurred in fewer than a third and for those who were obese in just over half. On the assumption that those not followed up had not changed, at the end of three years the proportion of patients with diastolic blood pressure $\geqslant 100 \mathrm{~mm} \mathrm{Hg}$ had fallen from 61 patients $(15.8 \%)$ to $31(8 \cdot 1 \%)$; the proportion of smokers had fallen from $103(26 \cdot 7 \%)$ to $94(24 \cdot 4 \%)$; and the proportion of obese patients had fallen from 87 $(22 \cdot 5 \%)$ to $79(20 \cdot 5 \%)$.

Conclusions-These changes were modest and in the absence of a control group cannot be attributed necessarily to health checks. Although the standard of follow up was better than in previously reported studies of the management of hypertension, the results emphasise the need to develop formal protocols for dietary and antismoking interventions and to evaluate formally the effectiveness (and cost effectiveness) of health checks.

\section{Introduction}

The Oxford prevention of heart attack and stroke project was set up as a feasibility study to test whether it was possible to implement the recommendation made in the Royal College of General Practitioners' report Prevention of Arterial Disease in General Practice that general practices should identify all patients who smoke, have high blood pressure, or are obese.' The role of a nurse facilitator in helping primary health care teams, particularly practice nurses, to develop their existing preventive role was explored, ${ }^{2}$ and the change in recording of the three risk factors after the introduction of opportunistic "health checks" was formally evaluated. ${ }^{3}$ As a result of this project the model of health checks has gained wide acceptance and 73 health authorities in the United Kingdom (with a catchment population of 24 million) have now appointed a "facilitator."

The royal college's recommendation was based on the knowledge that effective treatment of hypertension can reduce cerebrovascular and possibly cardiovascular disease ${ }^{4}$ and that antismoking advice by general practitioners can reduce the prevalence of smoking. ${ }^{5}$ The effectiveness of health checks administered by nurses in reducing risk, however, has not been directly assessed, and the results of an earlier study on screening for cardiovascular risk factors and intervention in British industry showed that a reduction in risk cannot be guaranteed. ${ }^{6}$ In view of the extent to which health checks in general practice are being promoted and adopted a randomised trial of their effectiveness, at least in reducing risk, is indicated. As it will be some years before the results of such a trial are known, however, it seemed sensible to address directly some of the anxieties that have been expressed about health checks. A key issue is the extent to which patients identified as at high risk of cardiovascular disease have been followed up. Recent audits of the follow up and management of hypertension in general practice are less than reassuring. ${ }^{8}$

As a first step in assessing the health checks carried out in Oxford a retrospective audit was made of all patients in whom raised blood pressure (diastolic blood pressure $\geqslant 90 \mathrm{~mm} \mathrm{Hg}$ or systolic blood pressure $\geqslant 160 \mathrm{~mm} \mathrm{Hg}$ ) had been diagnosed at health checks carried out during 1982-4 at the three initial practices recruited to the project. We report the extent to which these patients had been followed up, not only for blood pressure but also because of smoking habit and obesity, during the three years after their health check.

\section{Patients and methods}

The content of the health checks administered by nurses has been previously described. ${ }^{2}$ The figure illustrates the formal protocol for managing hypertensive patients. During 1982-4, 525 out of 2935 patients aged 35-64 attending for health checks in three separate practices were found to have initial blood pressure readings of $\geqslant 160 \mathrm{~mm} \mathrm{Hg}$ (systolic) or $\geqslant 90 \mathrm{~mm} \mathrm{Hg}$ (diastolic). All patients not already receiving treatment for hypertension were asked to attend for two further measurements. In 77 patients $(15 \%)$ the mean of the initial three readings fell below $160 / 90 \mathrm{~mm} \mathrm{Hg}$ and no short term follow up was deemed necessary (see figure). These patients were excluded from the subsequent audit. Of the remaining 448 patients (including those who did not return for 
two further readings), five had died and 57 could not be traced (presumed or known to have moved), leaving 386 patients $(86 \%)$ whose medical records were available for audit.

The notes were audited by two of us (AF and CM), both of whom had worked as part of the Oxford project's "rent an audit" team." Records were scrutinised from the day of the health check to exactly three years later. Every measurement of blood pressure or weight or mention of smoking state was noted

TABLE I-Three year follow up of patients for each risk factor according to initial risk state at health check

\begin{tabular}{|c|c|c|c|c|}
\hline & \multirow{2}{*}{$\begin{array}{c}\text { No } \\
\text { of } \\
\text { patients }\end{array}$} & \multirow{2}{*}{$\begin{array}{c}\text { No (\%) } \\
\text { followed up } \\
\text { at least once }\end{array}$} & \multicolumn{2}{|c|}{$\begin{array}{l}\text { Mean No of visits at which relevant } \\
\text { risk factor recorded in notes }\end{array}$} \\
\hline & & & To doctor & To nurse \\
\hline \multicolumn{5}{|c|}{ Diastolic blood pressure $(\mathrm{mm} \mathrm{Hg})$ : } \\
\hline$\leqslant 95$ & 197 & $181(92)$ & 0.9 & $2 \cdot 1$ \\
\hline $96-105$ & 147 & $135(92)$ & $1 \cdot 6$ & $3 \cdot 1$ \\
\hline$\geqslant 106$ & 42 & $42(100)$ & $1 \cdot 8$ & $4 \cdot 2$ \\
\hline \multicolumn{5}{|c|}{ Smoking habit ${ }^{\star}$ : } \\
\hline Never & 137 & $30(22)$ & $0 \cdot 2$ & $<0 \cdot 1$ \\
\hline Former & 141 & $25(18)$ & $0 \cdot 2$ & $0 \cdot 1$ \\
\hline Current & 100 & $50(50)$ & 0.7 & $1 \cdot 1$ \\
\hline \multicolumn{5}{|c|}{ Body mass indext: } \\
\hline$\leqslant 24 \cdot 9$ & 134 & $61(46)$ & $0 \cdot 8$ & 0.9 \\
\hline $25-29 \cdot 9$ & 165 & $94(57)$ & $1 \cdot 1$ & 1.8 \\
\hline$\geqslant 30$ & 87 & $67(77)$ & $3 \cdot 7$ & $3 \cdot 6$ \\
\hline
\end{tabular}

$\star$ Eight people were not classified.

tQuetelet's index $\left(\mathrm{kg} / \mathrm{m}^{2}\right)$.

TABLE II-Annual follow up in second and third years according to known risk state at beginning of each year

\begin{tabular}{|c|c|c|c|c|}
\hline & \multicolumn{2}{|c|}{ Second year } & \multicolumn{2}{|c|}{ Third year } \\
\hline & $\begin{array}{c}\text { No at beginning } \\
\text { of year }\end{array}$ & $\begin{array}{l}\text { No (\%) followed up } \\
\text { during year }\end{array}$ & $\begin{array}{c}\text { No at beginning } \\
\text { of year }\end{array}$ & $\begin{array}{l}\text { No (\%) followed up } \\
\text { during year }\end{array}$ \\
\hline \multicolumn{5}{|c|}{ Diastolic blood pressure $(\mathrm{mm} \mathrm{Hg})$ : } \\
\hline$\leqslant 95$ & 297 & $228(77)$ & 320 & $232(73)$ \\
\hline $96-105$ & 76 & $64(84)$ & 56 & $43(77)$ \\
\hline$\geqslant 106$ & 13 & $11(85)$ & 10 & $8(80)$ \\
\hline \multicolumn{5}{|l|}{ Smoking habit: } \\
\hline Smoker & 95 & $26(27)$ & 96 & $28(29)$ \\
\hline New ex-smoker & 9 & $5(56)$ & $11^{\star}$ & $7(64)$ \\
\hline Continuing non-smoker & 282 & $42(15)$ & 279 & $39(14)$ \\
\hline \multicolumn{5}{|l|}{ Body mass index: } \\
\hline$\leqslant 24.9$ & 138 & $43(31)$ & 141 & $45(32)$ \\
\hline $25-29 \cdot 9$ & 179 & $75(42)$ & 174 & $62(36)$ \\
\hline$\geqslant 30$ & 69 & $37(54)$ & 71 & $37(52)$ \\
\hline
\end{tabular}

*Includes two new "new ex-smokers" and nine ex-smokers from previous year.

TABLE III -Changes in blood pressure, smoking habit, and body mass index in patients followed up in second or third year, or both, excluding patients not followed up

\begin{tabular}{|c|c|c|c|c|c|c|c|c|}
\hline & \multicolumn{4}{|c|}{ Second year } & \multicolumn{4}{|c|}{ Third year } \\
\hline & $\begin{array}{c}\text { No of } \\
\text { patients }\end{array}$ & Initial & Final & $\begin{array}{c}\mathrm{p} \\
\text { Value }\end{array}$ & $\begin{array}{c}\text { No of } \\
\text { patients }\end{array}$ & Initial & Final & $\begin{array}{c}\mathrm{p} \\
\text { Value }\end{array}$ \\
\hline \multicolumn{9}{|l|}{ Blood pressure $(\mathrm{mm} \mathrm{Hg})$ : } \\
\hline Median diastolic & 301 & 94 & 89 & $<0.001$ & 283 & 94 & 88 & $<0.001$ \\
\hline Median systolic & 301 & 159 & 150 & $<0.001$ & 283 & 160 & 150 & $<0.001$ \\
\hline No $(\%)$ not smoking & 57 & $24(42)$ & $30(53)$ & NS & 56 & $24(43)$ & $29(52)$ & ) NS \\
\hline Mean body mass index $\left(\mathrm{kg} / \mathrm{m}^{2}\right)$ & 155 & $28 \cdot 2$ & $27 \cdot 6$ & $<0.01$ & 144 & $28 \cdot 2$ & $27 \cdot 7$ & $<0.01$ \\
\hline
\end{tabular}

TABLE IV - Blood pressure, smoking habit, and body mass index at end of each year, patients not followed up included on basis of their last measurement

\begin{tabular}{lcccc}
\hline & Initial & First year & Second year & Third year \\
\hline Blood pressure, all patients $(\mathrm{n}=386):$ & & & & \\
No(\%) with diastolic pressure $\geqslant 100 \mathrm{~mm} \mathrm{Hg}$ & $61(16)$ & $34(9)$ & $29(8)$ & $31(8)$ \\
$\quad$ Median diastolic (mm Hg) & 94 & 90 & 89 & 89 \\
$\quad$ Median systolic (mm Hg) & 159 & 153 & 151 & 151 \\
Blood pressure, no previous treatment (n=274): & & & & \\
$\quad$ No(\%) with diastolic pressure $\geqslant 100 \mathrm{~mm} \mathrm{Hg}$ & $49(18)$ & $23(8)$ & $18(7)$ & $22(8)$ \\
Median diastolic (mm Hg) & 93 & 91 & 89 & 88 \\
$\quad$ Median systolic (mm Hg) & 160 & 153 & 150 & 150 \\
Smoking habit, all patients: & $283(73)$ & $293(76)$ & $290(75)$ & $292(76)$ \\
$\quad$ No(\%) not smoking & & & & \\
Body mass index, all patients: & 26.3 & 25.9 & 26.0 & 26.0 \\
$\quad$ Mean body mass index $\left(\mathrm{kg} / \mathrm{m}^{2}\right)$ & $134(35)$ & $138(36)$ & $141(37)$ & $143(37)$ \\
No(\%)<24.9 & $87(23)$ & $69(18)$ & $71(18)$ & $79(21)$ \\
No(\%) $\geqslant 30.0$ & & & &
\end{tabular}

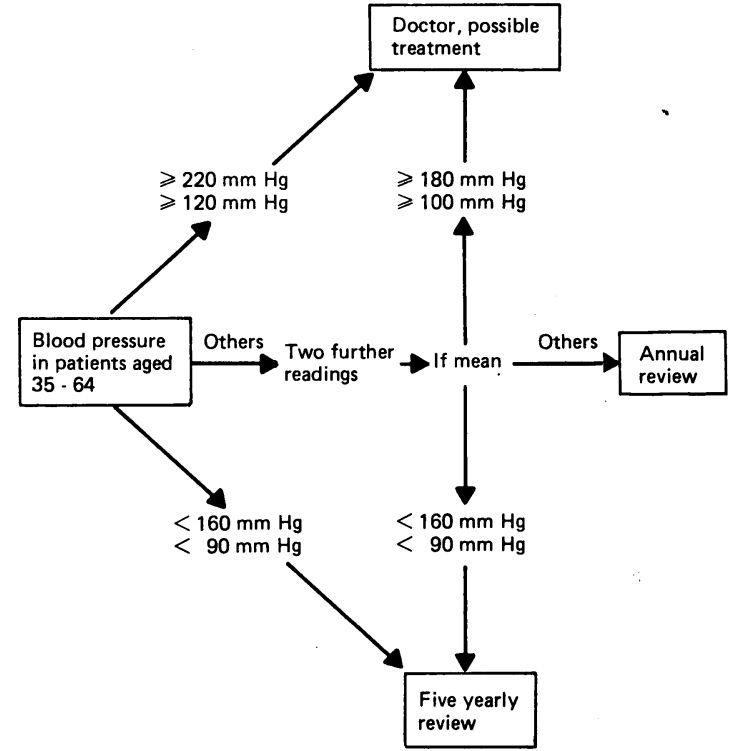

Protocol for nurses for managing blood pressure at health checks

chronologically, as was the status (doctor or nurse) of the person whom the patients consulted. In one surgery the nurse always wrote in a different colour ink, but in most cases the distinction between nurse and doctor was made on the basis of handwriting alone. Where separate records were kept by the nurse these were included in the audit. The total number of consultations during the three years was also noted.

Data were analysed on an AT microcomputer with DBASE to manipulate the data and the statistical package for the social sciences for the final analysis. The initial blood pressure reported in tables III and IV was assessed as the mean of up to three measurements made within six weeks after the health check and in table IV was based on three (192 patients), two (66), or one measurement (127). The initial body mass index and smoking habit reported in tables III and IV were those recorded at the health check except for eight patients in whom smoking state was first recorded at a later appointment during the first $\mathbf{4 2}$ days. The follow up blood pressures recorded in tables III and IV were based on the mean of the last three readings in the period or on the last two or on a single reading, as available. For table IV if no recordings had been made the blood pressure was taken to have remained unchanged since the previous period. For smoking habit and body mass index the measurements taken were the last recordings made. Following the example and reasoning of McAlister et $a l^{10}$ median values were preferred to means in the reporting of blood pressure. We used the McNemar test for paired dichotomous data, the Wilcoxon rank sum statistic, or the paired $t$ test, as appropriate, to generate the $\mathrm{p}$ values in table III.

\section{Results}

Table I shows the extent of follow up of patients according to their blood pressure, smoking habit, and weight at the health check. Within the three years after the health check, but excluding the initial six week assessment, nearly all patients (over 90\%) had their blood pressure rerecorded. Recorded follow up of smoking state in smokers and of weight in the obese, however, was less complete, with only half of smokers and 67 out of $87(77 \%)$ patients with a body mass index $>30$ having a further measurement recorded. Table I also gives some indication of the workload imposed by the follow up, though in the absence of a control group we cannot say to what extent this represents an addition to normal care. Consultations are reported for 
each risk factor individually; probably more than one risk factor was discussed at each consultation.

According to the protocol of the health check all patients in our audit should have been followed up at least once a year in the second and third years. Table II shows the extent to which this was achieved. As the necessity for follow up of smoking and obesity depends to some extent on response to advice, annual follow up in the second and third years after the initial health check is reported according to the patients' risk state at the beginning of each year. Whereas overall annual follow up of patients with diastolic blood pressure $>95 \mathrm{~mm} \mathrm{Hg}$ was about $80 \%$, for smoking habit in smokers it was less than $30 \%$, and for weight in the overweight about $35 \%$ and in the obese just over $50 \%$.

Table III presents the change in risk state in patients followed up in the second and third years. No data on a control population were available. A significant fall in blood pressure was evident, though some of this reduction must be attributed to regression to the mean and to patients becoming accustomed to having their blood pressure measured. In the 192 patients in whom the initial blood pressure was based on three readings the mean diastolic blood pressure at the health check was $98.0 \mathrm{~mm} \mathrm{Hg}$ and the mean of the first three measurements was $95.6 \mathrm{~mm} \mathrm{Hg}$. Although the proportion of smokers decreased, follow up was incomplete. In the entire audit only 21 patients reported a change in their smoking habit, with 14 stopping, five starting, and two stopping and then starting again. Of the 16 patients who reported stopping, only five did so for more than a year (documented on at least five occasions). The mean change in body mass index was also small despite reaching significance.

Table IV shows the overall achievement in reducing the cardiovascular risk of all patients who attended the initial health check. Patients without follow up were included on the assumption that they had not changed. The results show clearly that the overall impact on weight and smoking was limited.

\section{Discussion}

A major problem of assessing follow up by auditing medical records in general practice is that only a proportion of what takes place in the consultation is written down. This may well lead to an underestimation of the degree of follow up of smokers but probably not of weight or of blood pressure, for which objective measurements are performed. The method of calculating risk state used in table IV also makes for a conservative estimate of overall change (as patients not followed up were assumed not to have changed), but this does not seem unreasonable, and some patients lost to follow up of weight and blood pressure will undoubtedly have deteriorated.

Some of the reduction achieved in blood pressure must be attributed to patients becoming used to having their blood pressure measured: in the Framingham study the mean diastolic pressure for the whole population fell from 83.1 to $81.4 \mathrm{~mm} \mathrm{Hg}$ between the first and fourth examinations. ${ }^{11}$ Also the audit was only of patients identified as having raised blood pressure, for whom a formal protocol existed for follow up. Probably follow up of patients for obesity or smoking alone, for whom no protocol for follow up existed, would be worse.
In view of the appalling impact of smoking on so many aspects of health, and the demonstration by the Medical Research Council's mild hypertension trial that the difference in outcome between smokers and non-smokers was greater than between treatment 'groups, ${ }^{12}$ the apparent poor follow up of smokers (and lack of reported stopping smoking) is disappointing. Similarly, the balance of evidence suggests that reduction of weight has an important role in managing hypertension, ${ }^{13}$ though the extent of follow up and documented change is again unimpressive. In comparison with previous audits, however, the extent of adherence to protocol seems remarkably good - for example, Kurji and Haines reported that $69 \%$ of newly diagnosed hypertensives had no further recording measured within 12 months. ${ }^{7}$ Similarly, as recently as 1986 Stern reported from 12 Oxfordshire practices that smoking habit was recorded in only $44 \%$ of the notes of hypertensive patients and weight in $31 \% .^{14}$

These observations make a case for including nurses in managing chronic disease at least as much as for health checks. Arguably, health checks are screening tests and should not be widely promoted until their effectiveness has been shown in a randomised trial. Our results provide substantive arguments in favour of this view. As stated by Knox in 1974, it remains inappropriate to screen for risk factors if follow up (and ultimately reduction in risk and mortality) cannot be achieved. ${ }^{15}$ At this stage, however, the quality of health checks and their follow up should be improved rather than them being abandoned.

This audit shows that good follow up of blood pressure and possibly a reduction in the number of hypertensive patients can be achieved if enthusiasm is maintained by a nurse facilitator. In future, considerable attention needs to be given to stopping smoking and reducing weight. Protocols similar to those drawn up for managing hypertension are required for those who smoke and for the obese. Unfortunately, at the present time there is little evidence of effective practice on which such protocols, particularly for dietary intervention, could be based.

1 Royal College of General Practitioners. Prevention of arterial disease in general practice. London: RCGP, 1981. (Report from General Practice No 20.)

2 Fullard E, Fowler G, Gray JAM. Facilitating prevention in primary care. BrMed f 1984;289:1585-7.

3 Fullard E, Fowler G, Gray JAM. Promoting prevention in primary care: controlled trial of low technology, low cost approach. $\mathrm{Br} M e d \mathcal{F}$ 1987;294: control 1080 .

4 Wilcox RG, Mitchell JRA, Hampton JR. Treatment of high blood pressure: should clinical practice be based on the results of clinical trials. Br Med $\mathcal{F}$ 1986;293:433-7.

5 Russell MAH, Wilson C, Taylor C, Baker CD. Effect of general practitioners' advice against smoking. Br Med f 1979;ii:231-5.

6 Rose G, Heller RF, Tunstall-Pedoe $\mathrm{H}$, et al. Heart disease preventative project: a randomised controlled trial in British industry. $\mathrm{Br} \mathrm{Med} \mathrm{f}$ $1980 ; 280: 741-51$

$7 \mathrm{Kurji}$ K, Haines A. Detection and management of hypertension in general practices in north west London. Br Med F 1984;288:903-6.

8 Michael G. Quality of care in managing hypertension by case finding in north west London. BrMed f 1984;288:906-8.

9 Gray JAM, O'Dwyer MA, Fullard EM, Fowler GH. Rent-an-audit. $f R$ Coll Gen Pract 1987;37:177.

10 McAlister NH, Coney HD, Tong C, Lee A, Wigle ED. Randomised controlled trial of computer assisted management of hypertension in general practice. BrMed f 1986;293:670-4.

11 Dawber TR. The Framingham study. Cambridge, Massachusetts: Harvard University Press, 1980.

12 Medical Research Council Working Party. MRC trial of treatment of mild hypertension: principal results. Br Med f 1985;291:97-104.

13 Anonymous. Weight reduction in hypertension [Editorial]. Lancet 1985; ;: $1251-2$.

14 Stern D. Management of hypertension in 12 Oxfordshire general practices. $f R$ Coll Gen Pract 1986;36:549-51.

15 Knox EG. Screening for disease. Lancet 1974;ii: 1434-6.

(Accepted 30 March 1989) 